\title{
Nucleon polarised parton distribution functions
}

\author{
Gerhard K. Mallot \\ CERN, 1211 Geneva 23, Switzerland
}

\begin{abstract}
An introduction to the present status of spin-dependent parton distribution functions is given. Both, results from lepton scattering experiments and from polarised proton-proton collisions with longitudinal polarisation are included. Particular emphasis is put on recent results for the gluon polarisation, which is a key element in the nucleon spin structure. Very large gluon polarisation scenarios, put forward to explain the small quark spin contribution to the nucleon spin, are now strongly disfavoured.
\end{abstract}

Key words: PDF, deep inelastic scattering, pp collisions, polarization PACS: 13.60.-r, 13.85.Ni, 13.88.+e, 14.20.Dh, 14.65.-q, 21.10.Hw

\section{Introduction}

The cross-sections of many hard QCD processes are known to factorise into a calculable partonic part and universal parton distribution functions (PDFs), which contain the information on the internal structure of the interacting objects in terms of quarks and gluons. The longitudinal spin structure, discussed here, requires the measurement of double-spin cross-section asymmetries. Inclusive deep inelastic scattering (DIS) of leptons on nucleons is the cleanest tool to study these distributions. In addition to DIS experiments on protons and neutrons (deuteron, helium-3), semi-inclusive DIS (SIDIS) experiments can help to clarify the contributions of the individual quark flavours. The detected hadron in the final state tags to some extent the flavour of the quark in the underlying hard interaction. Unlike in the unpolarised case (HERA) a polarised leptonnucleon collider does not exist. Thus the accessible kinematic range in the small $x$-Bjorken region is presently determined by the DIS experiments with muons at CERN (COMPASS , SMC, EMC) to $x>0.003$ with a 4-momentum transfer $Q^{2}>1 \mathrm{GeV}^{2}$. Very precise data for $x>0.03$ come from experiments at SlaC and Hermes at Desy and from Jlab for large $x$.

Polarised $p p$ collisions at RHIC have opened a new source of information on polarised PDFs. Unlike in DIS here the underlying process is the interactions of two partons 

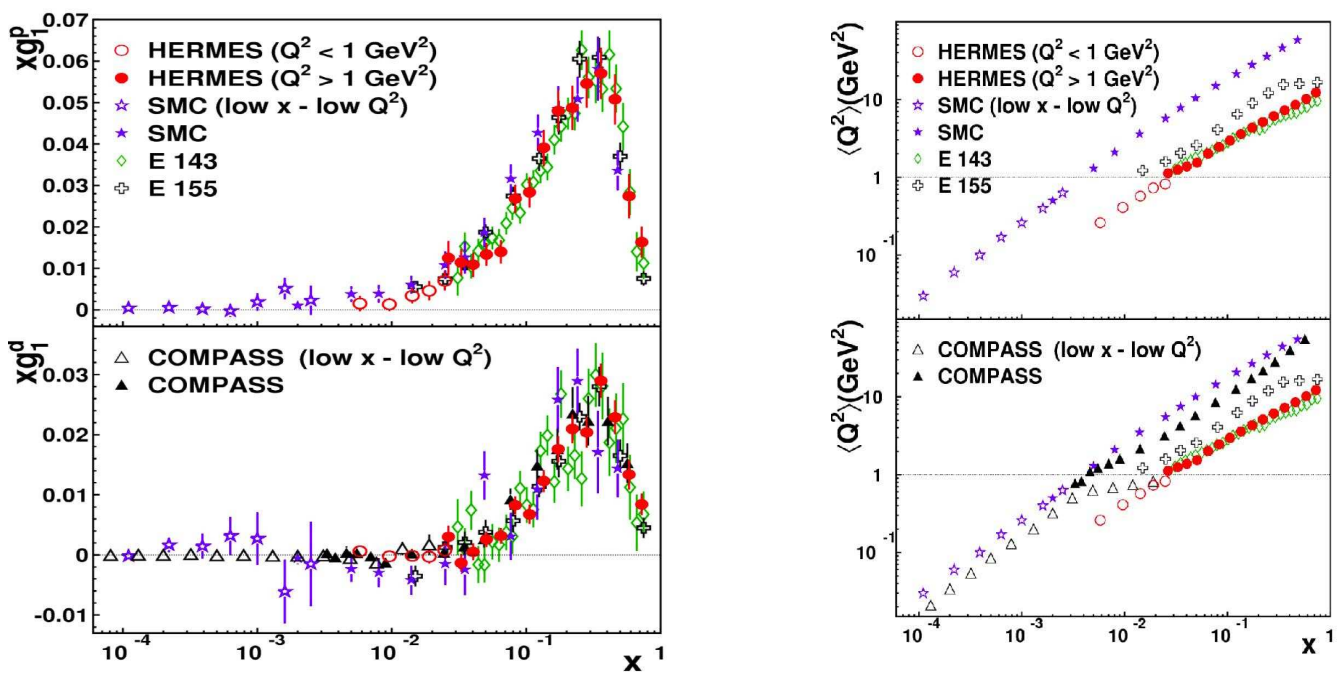

Fig. 1. Summary of $g_{1}$ proton (top) and deuteron (bottom) world data (left) and $Q^{2}$ ranges (right) (from $[2])$.

(quarks, gluons). Thus two PDFs are involved in the process. Most data up to now come from neutral pion production where like in SIDIS the knowledge of fragmentation functions (FF) is needed to interpret the data in terms of PDFs. Neutral and charged current interactions will yield excellent data after the RHIC upgrade to a centre-of-mass energy of $500 \mathrm{GeV}$. The planned electron-icon collider EIC at RHIC (or JLAB) ensures a long-term perspective in the direct study of PDFs.

A central question in nucleon spin physics is whether the gluon polarisation is large enough to understand the observed smallness of the axial charge $a_{0}$ (spin crisis) via the axial anomaly. The COMPAss and RHIC data show that this scenario is very much disfavoured. The gluon polarisation in the region $x_{g} \simeq 0.1$ is very small and compatible with zero. Although the first moment $\Delta G$ of the gluon distribution function cannot be determined model independently from the present measurements, large values of $\Delta G$ are difficult to bring into agreement with present data.

Of central importance in the study of PDFs are global analyses of all data. Recent attempts show the strength of these methods.

\section{Inclusive DIS}

The discovery by the European Muon Collaboration [6] that the first moment $\Gamma_{1}$ of the spin-dependent structure function $g_{1}$

$$
\Gamma_{1}^{p / n}\left(Q^{2}\right)=\int_{0}^{1} \mathrm{~d} x g_{1}^{p / n}\left(x, Q^{2}\right)=\frac{1}{36}\left(4 \Delta \Sigma \pm 3 \Delta q_{3}+\Delta q_{8}\right)\left(1+\frac{\alpha_{s}}{\pi}\right)+\mathcal{O}\left(\alpha_{s}^{2}\right)
$$

of the proton is much smaller than expected implies that the total contribution of the quark spins to the nucleon spin $\Delta \Sigma \equiv \Delta u+\Delta d+\Delta s$ is small. Here $\Delta q=\left(q_{+}-q_{-}\right)+$ $\left(\bar{q}_{+}-\bar{q}_{-}\right)$is the difference of the number of quarks and antiquarks of flavour $q$ with 




Fig. 2. Non-strange and strange quark helicity distributions $\Delta q$ as a function of $x$ from CoMPASS [3]. Full and open spheres correspond to the DSS and EMC fragmentation functions, respectively.

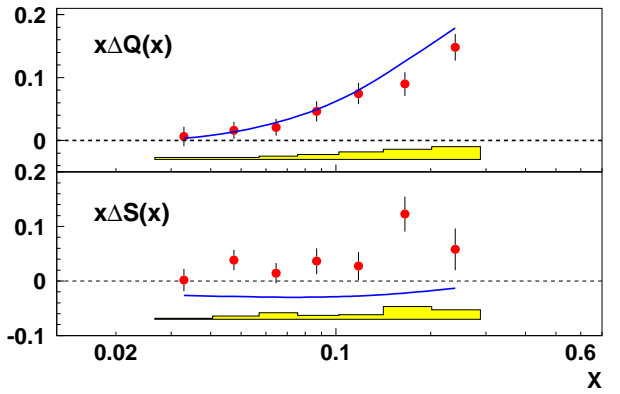

Fig. 3. Top: Non-strange and strange quark helicity distributions $(\Delta Q=\Delta q+\Delta \bar{q})$ at $Q_{0}^{2}=2.5 \mathrm{GeV}^{2}$ as a function of Bjorken $x$ from Hermes [4]. The curves represent an earlier analysis of world data [5].

positive and negative helicity and $\Delta q_{3} \equiv \Delta u-\Delta d$ and $\Delta q_{8} \equiv \Delta u+\Delta d-2 \Delta s$ are known from $\beta$ decays.

The world data on the structure function $g_{1}$ for the proton and the deuteron are summarised in Fig. 1. In addition to the data shown, CLAS at JLab collected very precise proton and deuteron $g_{1}$ data covering the range $0.05<Q^{2}<5 \mathrm{GeV}^{2}$ [7]. However, for $Q^{2}>1 \mathrm{GeV}^{2}$ the range is reduced to $0.15<x<0.58$. A new experiment. SANE in JLAB Hall $\mathrm{C}$, will investigate the proton spin structure in the range $0.3<x<0.8$ [8].

\section{Semi-inclusive DIS}

Both, the Hermes and Compass Collaboration evaluated $\Delta \Sigma$ from their deuteron data with $Q^{2}>1 \mathrm{GeV}^{2}$ yielding $\Delta \Sigma=0.330 \pm 0.025$ (exp.) \pm 0.028 (evol.) \pm 0.011 (theo.) at $Q^{2}=5 \mathrm{GeV}^{2}$ from HERMES and $\Delta \Sigma=0.35 \pm 0.03$ (stat.) \pm 0.05 (syst.) at $Q^{2}=3 \mathrm{GeV}^{2}$ from Compass. The results are in excellent agreement. The value for $\Delta \Sigma$ is somewhat larger than the original EMC result of $\Delta \Sigma=0.12 \pm 0.17$, which was given at a larger scale $Q^{2}=10.7 \mathrm{GeV}^{2}$. All results are consistent with each other upon taking evolution into account. Therefore, the conclusion that the quark spins contribute little to the nucleon spin remains valid.

This violation of the Ellis-Jaffe sum rule requires the first moment of the strange sea $\Gamma_{1}^{s}$ to be negative. More direct information on the strange sea can be obtained from semiinclusive DIS, in particular from data with identified kaons. The Hermes and Compass Collaborations analysed their data including charged kaon asymmetries in leading-order QCD in terms of the truncated first moments $\left.\Gamma_{1}\right|_{x}$ calculated in the measured $x$-Bjorken range. HeRmes obtains a positive value of $\left.\Gamma_{1}^{s+\bar{s}}\right|_{x}=0.037 \pm 0.019 \pm 0.027$ at $Q^{2}=$ $2.5 \mathrm{GeV}^{2}$ for $0.02<x<0.6$ (Fig. 3). Compass finds for a larger range $0.004<x<0.3$ a slightly negative value $\left.\Gamma_{1}^{s}\right|_{x}=-0.01 \pm 0.01 \pm 0.01$ at $Q^{2}=3 \mathrm{GeV}^{2}$ (Fig. 2). The contribution from the unmeasured region $x>0.3$ is negligible. The results depend crucially on the size of the contributions of the $\bar{s}$ quark and the $u$ quark to the fragmentation 


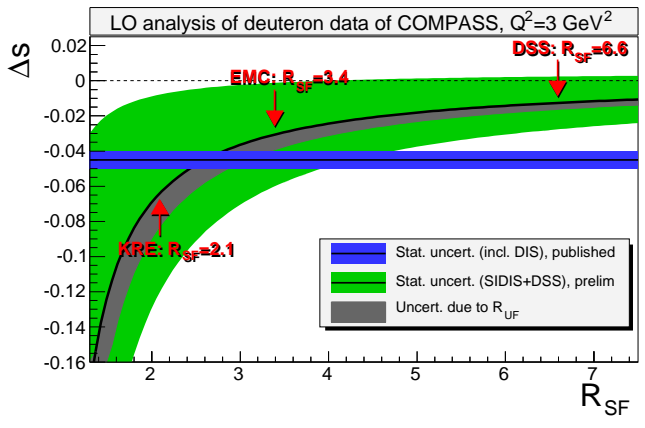

Fig. 4. Truncated first moment of $\Delta s$ as function of the ratio of strange to up quark fragmentation to kaons $R_{S F}$ [3]. The most realistic value $R_{S F}=6.6$ is the one from the DSS analysis [9]. For small $s$ to $K$ fragmentation the error on $\Gamma_{1}^{s}$ would become very large.

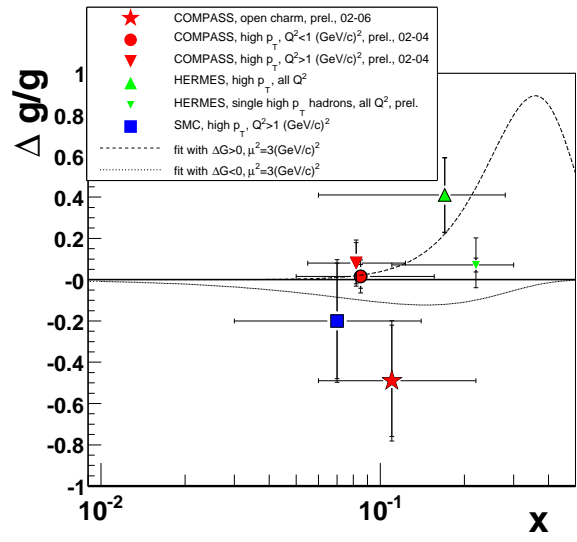

Fig. 5. Gluon polarisation $\Delta g / g$ as function of $x_{g}$ obtained from LO PGF analysis of SIDIS data. The curves show two solutions of a NLO QCD analysis by Compass [11].

into positive kaons, characterised by the ratio $R_{S F}=\int D_{\bar{s}}^{K^{+}}(z) \mathrm{d} z / \int D_{u}^{K^{+}}(z) \mathrm{d} z$ (and charge conjugate). This is demonstrated in Fig. 2 by a comparison to an analysis using the historical FF assumed by the EMC. An analysis of $\left.\Gamma_{1}^{s}\right|_{x}$ with $R_{S F}$ as parameter was performed by Compass [3] using the cross-section weighted sum of the charged kaon asymmetries $A^{K^{+}+K^{-}}$. The value and the size of the error of $\left.\Gamma_{1}^{s}\right|_{x}$ strongly depend on the value of $R_{S F}$ (Fig. 4). A recent evaluation of the fragmentation functions [9] favours a value of $R_{S F}=6.6$ which is in line with the FFs used in the HERMES analysis.

\section{Gluon Polarisation}

Apart from the contribution of the quark spins $\Delta \Sigma$, the nucleon spin sum rule

$$
\frac{1}{2}=\frac{1}{2} \Delta \Sigma+\Delta G+L_{z}
$$

receives contributions from gluon spins $\Delta G$ and from orbital angular momentum $L_{z}$, which must compensate for the smallness of $\Delta \Sigma$. Experiments start to obtain information on the gluon polarisation $\Delta G$. The gluon polarisation can be studied in polarised DIS and SIDIS and in $\vec{p} \vec{p}$ interactions.

The gluon polarisation enters directly in photon-gluon fusion (PGF) processes $\gamma g \rightarrow$ $q \bar{q}$ in which a quark-antiquark pair is produced. In SIDIS it gives rise to a double-spin cross-section asymmetry

$$
A_{\|}=R_{\mathrm{pgf}} a_{L L}^{\mathrm{pgf}} \frac{\Delta G}{G}+A_{\mathrm{bgd}},
$$

where $R_{\text {pgf }}$ is the fraction of PGF events and $a_{L L}^{\mathrm{pgf}}$ is the analysing power of the PGF subprocess. Until now all analyses of such data were performed in leading order. For a particular measurement both, $R_{\mathrm{pgf}}$ and the average $a_{L L}^{\mathrm{pgf}}$, have to be estimated using Monte Carlo (MC) simulations. This introduces a model dependence in the determination of $\Delta G / G$. In the light-quark case the QCD-Compton process $\gamma q \rightarrow q g$ and the direct 


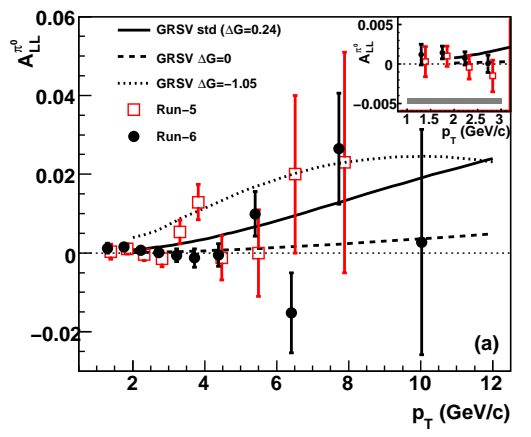

Fig. 6. Phenix inclusive $\pi^{0}$ asymmetries $A_{L L}$ as function of $p_{T}$ [13]. The curves correspond to the NLO predictions for various GRSV parameterisations [15].

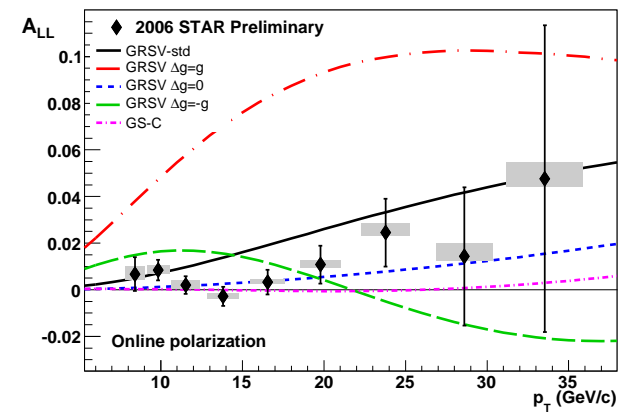

Fig. 7. Star inclusive jet asymmetries $A_{L L}$ as function of $p_{T}$ [14]. The curves correspond to the NLO predictions for various GRSV parameterisations [15].

process $\gamma q \rightarrow q$ limit $R_{\text {pgf }}$ to about $30 \%$, while for charmed quark pairs $R_{\text {pgf }}$ is essentially unity. Here the challenges are the low production cross-section and the detection of open charm ( $D$ mesons).

HERMES determined $\Delta G / G$ from single high- $p_{T}$ hadron production asymmetries in four bins of transverse hadron momentum $p_{T}$ in the range $1.05 \mathrm{GeV}<p_{T}<2.5 \mathrm{GeV}$ [10]. The resulting gluon polarisation is small $\Delta G / G=0.071 \pm 0.034$ (stat.) \pm 0.010 (syst.) ${ }_{-0.105}^{+0.127}$ (model) and probed around $x_{g} \simeq 0.22$ at $\mu^{2}=1.35 \mathrm{GeV}^{2}$.

Compass determined $\Delta G / G$ from the cross-section asymmetries for $D$ meson production [11]. This method relies much less on Monte Carlo simulations but is limited in statistical precision. A neural network was used to estimate $a_{L L}^{\mathrm{pgf}}$ from the event kinematics on an event-by-event basis. The result from the $2002-2006$ data set is $\Delta G / G=$ $-0.49 \pm 0.27 \pm 0.11$ (syst.) compatible with zero. The gluon distribution is probed around $x_{g}=0.11$ at $\mu^{2}=13 \mathrm{GeV}^{2}$. This is also compatible with the most precise Compass result from light-quark pairs at $Q^{2}<1 \mathrm{GeV}^{2}$ of $\Delta G / G=0.016 \pm 0.058 \pm 0.055$ (syst.). All results from PGF in DIS are summarised in Fig. 5.

At RHIC cross-section asymmetries for longitudinally polarised $\vec{p} \vec{p}$ scattering at $\sqrt{s}=$ $200 \mathrm{GeV}$ were analysed for several channels, in particular inclusive $\pi^{0}$ production at PHENIX [12,13] and inclusive jet production at STAR [14] (Figs. 6,7). The cross-sections are well understood over seven orders of magnitude in NLO. The data are hardly compatible with the standard scenario PDFs of GRSV [15] and favour small or even negative gluon polarisation. Due to the involvement of partons from two nucleons the cross-section is a convolution over a large range in $x_{g}$ from about 0.01 to almost 1 with a mean value of $\langle x\rangle \simeq 2 p_{T} / \sqrt{s}$. PHENIX quotes a gluon $x$ range of 0.02 to 0.3 [12] for the measured $p_{T}$ range. Future measurements at $\sqrt{s}=500 \mathrm{GeV}$ will remove the present sign ambiguity because of the decreasing relative importance of the quadratic term in $\Delta G$ with increasing $p_{T}$. Data taken at $\sqrt{s}=62.4 \mathrm{GeV}$ allow to probe higher $x_{g}$.

\section{Global analyses}

Global QCD analyses of inclusive data in NLO are a well known tool. Recently also semi-inclusive data and the RHIC $p p$ data were included in these analyses. However, the 


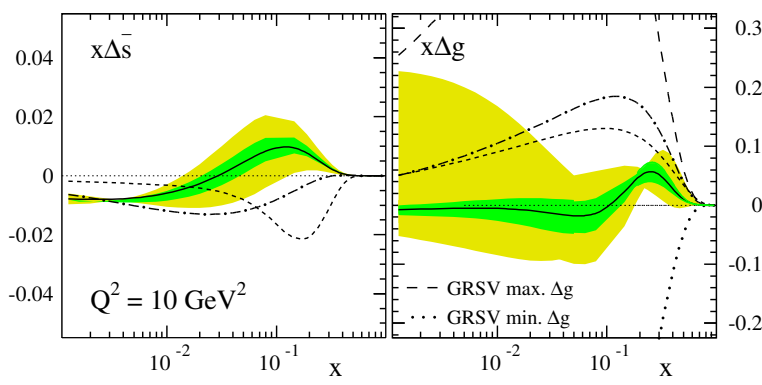

Fig. 8. Spin-dependent PDFs from the DSSV analysis [16] of DIS, SIDIS and $p p$ data as function of $x$. Left: strange quark distribution; right: gluon distribution. The darker, inner and the lighter, outer uncertainty band correspond to an increase of $\Delta \chi^{2}=1$ and of $\Delta \chi^{2} / \chi^{2}=2 \%$, respectively.

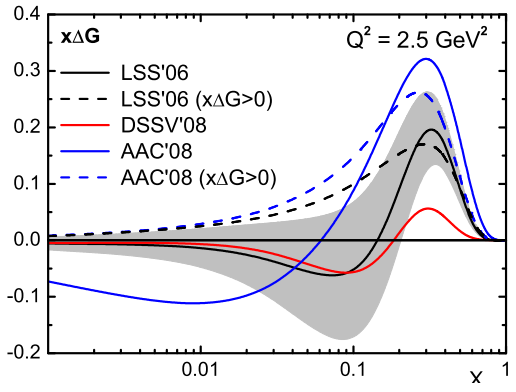

Fig. 9. Comparison of spin-dependent gluon PDFs from various global analyses [17].

asymmetries for hadron pairs are not yet included due to the difficulty to describe them in NLO. The maybe most advanced analysis (DSSV, [16]) is shown in Fig. 8. Interesting is the node is the gluon polarisation $\Delta g$ around $x_{g}=0.1$. Clearly visible is the influence of the RHIC data on the error in the range $0.05<x_{g}<0.2$. The strange quark polarisation $\Delta s$ is positive at larger $x$ due to the HERMES semi-inclusive data but the integral is slightly negative. The CompAss kaon asymmetries are not yet used in the analysis. A comparison of $\Delta g(x)$ from various global analyses are compared in Fig. 9. All analyses find rather small gluon polarisations, however its first moment could still significantly contribute to the nucleon spin.

\section{References}

[1] Slides: http://www.weizmann.ac.il/MaKaC/ materialDisplay . py?contribId=331\&sessionId=23\&materialld=slides\&conf $I d=0$

[2] L. de Nardo, Circum-Pan-Pacific Symposium on High-Energy Spin Physics 2007, Vancouver, CN, 30 July-August 2, 2007.

[3] R. Windmolders et al. [COMPASs], SPIN 2008, Charlottesville, VA, USA, October 2008.

[4] A. Airapetian et al. [Hermes], Phys. Lett. B 666 (2008) 446

[5] E. Leader, A. V. Sidorov and D. B. Stamenov, Phys. Rev. D 73 (2006) 034023.

[6] J. Ashman et al. [EMC], Nucl. Phys. B 328, 1 (1989).

[7] K. V. Dharmawardane et al. [CLAS], Phys. Lett. B 641, 11 (2006).

[8] S. Wood, these proceedings.

[9] D. de Florian, R. Sassot and M. Stratmann, Phys. Rev. D 75 (2007) 114010.

[10] P. Liebing, "Determination of $\Delta g / g$ from Hermes Data on High- $p_{T}$ Inclusive Charged Hadrons", Proc. of 15th Int. Workshop on Deep-Inelastic Scattering and Related Subjects, Munich, April 2007, http://dx.doi.org/10.3360/dis.2007.83

[11] J. Nassalski, these proceedings.

[12] A. Bazilevsky, these proceedings.

[13] A. Adare et al. [PHENIX Collaboration], [arXiv:0810.0694].

[14] B. Surrow, these proceedings.

[15] M. Glück, E. Reya, M. Stratmann and W. Vogelsang, Phys. Rev. D 63, 094005 (2001).

[16] D. de Florian, R. Sassot, M. Stratmann and W. Vogelsang, Phys. Rev. Lett. 101 (2008) 072001 [arXiv:0804.0422].

[17] S. E. Kuhn, J. P. Chen and E. Leader, arXiv:0812.3535 [hep-ph]. 